\section{Genome Sequence Resource of Phytophthora colocasiae from China Using Nanopore Sequencing Technology}

Zhixin Wang, ${ }^{1}$ Jiandong Bao, ${ }^{2}$ Lin Lv, ${ }^{1}$ Lianyu Lin, ${ }^{2}$ Zhiting Li, ${ }^{1}$ Mingyue Shi, ${ }^{3}$ Yuting Huang, ${ }^{3}$ Rongbo Wang, ${ }^{3}$ Benjin Li, ${ }^{3}$ Peiqing Liu, ${ }^{3}$ and Qinghe Chen ${ }^{1,3, \dagger}$

${ }^{1}$ Key Laboratory of Green Prevention and Control of Tropical Plant Diseases and Pests, Ministry of Education, College of Plant Protection, Hainan University, Haikou 570228, China

${ }^{2}$ Fujian University Key Laboratory for Plant-Microbe Interaction, College of Life Sciences, Fujian Agriculture and Forestry University, Fuzhou, 350002, China

${ }^{3}$ Fujian Key Laboratory for Monitoring and Integrated Management of Crop Pests, Institute of Plant Protection, Fujian Academy of Agricultural Sciences, Fuzhou 350003, China

\begin{abstract}
Phytophthora colocasiae is a destructive oomycete pathogen of taro (Colocasia esculenta), which causes taro leaf blight. To date, only one highly fragmented Illumina short-read-based genome assembly is available for this species. To address this problem, we sequenced strain Lyd2019 from China using Oxford Nanopore Technologies long-read sequencing and Illumina short-read sequencing. We generated a $92.51-\mathrm{Mb}$ genome assembly consisting of 105 contigs with an $\mathrm{N}_{50}$ of $1.70 \mathrm{Mb}$ and a maximum length of $4.17 \mathrm{Mb}$. In the genome assembly, we identified $52.78 \%$ repeats and 18,322 protein-coding genes, of which 12,782 genes were annotated. We also identified 191 candidate RXLR effectors and 1 candidate crinkling and necrosis effector. The updated near-chromosome genome assembly and annotation resources will provide a better understanding of the infection mechanisms of P. colocasiae.
\end{abstract}

\section{Genome Announcement}

Taro (Colocasia esculenta (L.) Schott) is an increasingly important crop used as a vegetable, staple food, and traditional medicine in Asia, Africa, South America, and the Caribbean and Pacific islands (Kreike et al. 2004).

Taro leaf blight, caused by Phytophthora colocasiae Racib., is the most destructive disease of taro (Raciborski 1900); it has become a major constraint on taro production in all taro-growing countries, causing yield losses of 25 to 30\% (Jackson et al. 1980; Mbong et al. 2013). The pathogen also causes serious postharvest corm decay, which leads to heavy storage losses (Mbong et al. 2015). Taro leaf blight symptoms appear as small, water-soaked spots that increase in size and number over time. Under cloudy weather conditions, with intermittent rain and temperatures of approximately $28^{\circ} \mathrm{C}$, the disease quickly spreads across entire fields, giving them a blighted appearance (Mishra et al. 2008). Heavily infected taro fields in locations such as Southeast Asia have experienced crop losses of more than 30\% (Singh et al. 2012; Vasquez 1990). Therefore, appropriate and effective control strategies are urgently needed.

Genome data represent a valuable resource for the identification and elucidation of pathogenesis mechanisms. Several genome sequences for plant-pathogenic oomycetes are now available, and much of the current understanding of the mechanisms and evolution of the virulence in oomycetes has been based on the genome and transcriptome sequencing efforts

\footnotetext{
${ }^{\dagger}$ Corresponding author: Q. Chen; qhchen@ hainanu.edu.cn
}

\section{Z. Wang and J. Bao contributed equally to this work.}

The author(s) declare no conflict of interest.

Accepted for publication 10 May 2021.

\section{Funding}

This work was supported by grants from the National Natural Science Foundation of China (31772141), the Scientific Research Foundation of Hainan University (KYQD(ZR)-20080), and the Innovation Team of Plant Protection, Fujian Academy of Agricultural Sciences (STIT2017-1-8).

\section{Keywords}

genomics, pathogenicity, Phytophthora colocasiae, taro leaf blight 
Table 1. Genome characteristics

\begin{tabular}{|c|c|}
\hline Features & Strain Lyd2019 \\
\hline ONT long reads ${ }^{a}$ & $11.78 \mathrm{~Gb}$ (approximately $127 x$ ) \\
\hline Average read length & $13.15 \mathrm{~kb}$ \\
\hline Reads $\mathrm{N}_{50}$ & $16.85 \mathrm{~kb}$ \\
\hline Maximum read length & $176 \mathrm{~kb}$ \\
\hline Illumina short reads & 4.56 Gb (approximately 49x) \\
\hline Estimated genome size ${ }^{\mathrm{b}}$ & $92.30 \mathrm{Mb}$ \\
\hline Estimated genome repeats ${ }^{\mathrm{b}}$ & $56.71 \%$ \\
\hline Estimated genome heterozygosity ${ }^{\mathrm{b}}$ & $0.28 \%$ \\
\hline Assembly size & $92.51 \mathrm{Mb}$ \\
\hline Contig number & 105 \\
\hline Contig $\mathrm{N}_{50}$ & $1.70 \mathrm{Mb}$ \\
\hline Contig $L_{50}$ & 20 \\
\hline Average contig length & $0.88 \mathrm{Mb}$ \\
\hline Maximum contig length & 4.17 Mb \\
\hline GC content & $51.05 \%$ \\
\hline Repeat sequence & $52.78 \%$ \\
\hline Illumina reads mapping rate & $96.67 \%$ \\
\hline Protein-coding genes & 18,322 \\
\hline Candidate RXLR effectors & 191 \\
\hline Candidate CRN effectors ${ }^{c}$ & 1 \\
\hline Genes annotated by Pfam ${ }^{c}$ & 11,538 \\
\hline Genes annotated by $\mathrm{GO}^{\mathrm{c}}$ & 4,375 \\
\hline Genes annotated by KEGG ${ }^{c}$ & 6,857 \\
\hline Genes annotated by $\mathrm{KOG}^{\mathrm{C}}$ & 11,382 \\
\hline Genes annotated by CAZy ${ }^{\mathrm{c}}$ & 335 \\
\hline Total of protein-coding genes annotated & 12,782 \\
\hline \multicolumn{2}{|c|}{ 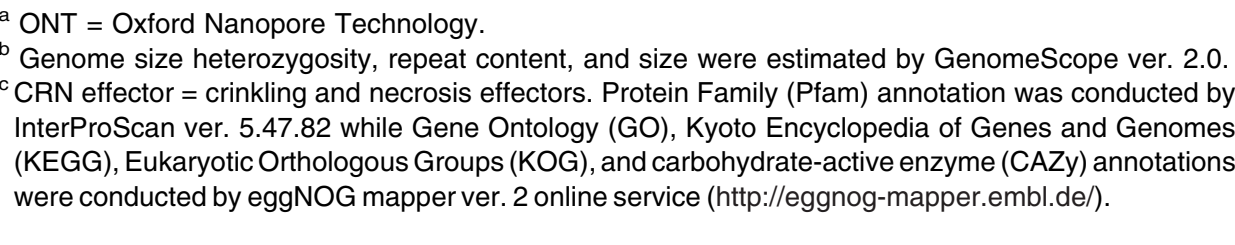 } \\
\hline
\end{tabular}

(Lee et al. 2020; Srivastava et al. 2020). A recent study reported an Illumina short-read-based $P$. colocasiae genome assembly of strain 7290 from Vietnam (Vetukuri et al. 2018). The genome assembly was $56.6 \mathrm{Mb}$ in size and consisted of 19,853 scaffolds with an $\mathrm{N}_{50}$ of $11.26 \mathrm{~kb}$ and maximum length of $136.77 \mathrm{~kb}$ (Vetukuri et al. 2018). The genome assembly contained 19,984 predicted genes, among which 11,200 were annotated using the Protein Family (Pfam) database; 337 genes were found to encode candidate RXLR effectors (Vetukuri et al. 2018). These resources provide fundamental $P$. colocasiae genomic information; however, the reported genome assembly was highly fragmented and the scaffold $\mathrm{N}_{50}$ was low, indicating poor quality of the genome assembly and limiting its downstream applications such as gene synteny analysis.

To address this limitation, high-quality genome assembly of $P$. colocasiae is urgently needed. We collected $P$. colocasiae strain Lyd2019 from China and its mycelia were sent to Biomarker Technologies Corp. (Beijing, China) for DNA extraction, genomic library construction, and whole-genome sequencing. We obtained $11.78 \mathrm{~Gb}$ (approximately $127 \times ; \mathrm{N}_{50}=$ $16.85 \mathrm{~kb}$ ) of long reads using the Oxford Nanopore Technologies (ONT) PromethlON sequencing platform, and $4.56 \mathrm{~Gb}$ (approximately 49x) of 150-bp paired-end short reads (PE150) using the Illumina HiSeq 3000 sequencing platform (Table 1).

Genome size estimation was conducted using GenomeScope ver. 2.0 (Ranallo-Benavidez et al. 2020) based on the k-mer distribution of the lllumina short reads ( $k=25$; diploid model). The results showed a main peak at a depth of 37 , which was the average k-mer depth. The estimated genome size was $92.30 \mathrm{Mb}$, the estimated proportion of repeats was $56.71 \%$, and the estimated heterozygosity was $0.28 \%$ (Table 1 ; Fig. $1 \mathrm{~A}$ ).

We used NextDenovo ver. 2.3.1 (https://github.com/Nextomics), a string-graph-based de novo assembler, to generate a draft genome assembly using only ONT long reads (seed reads were cut off at 21,827 bp). Then, we used NextPolish ver. 1.3.0 (https://github.com/Nextomics) to correct base errors in the draft genome assembly using both ONT long reads and Illumina short reads. Finally, we obtained a $92.51-\mathrm{Mb}$ polished genome assembly consisting of 105 


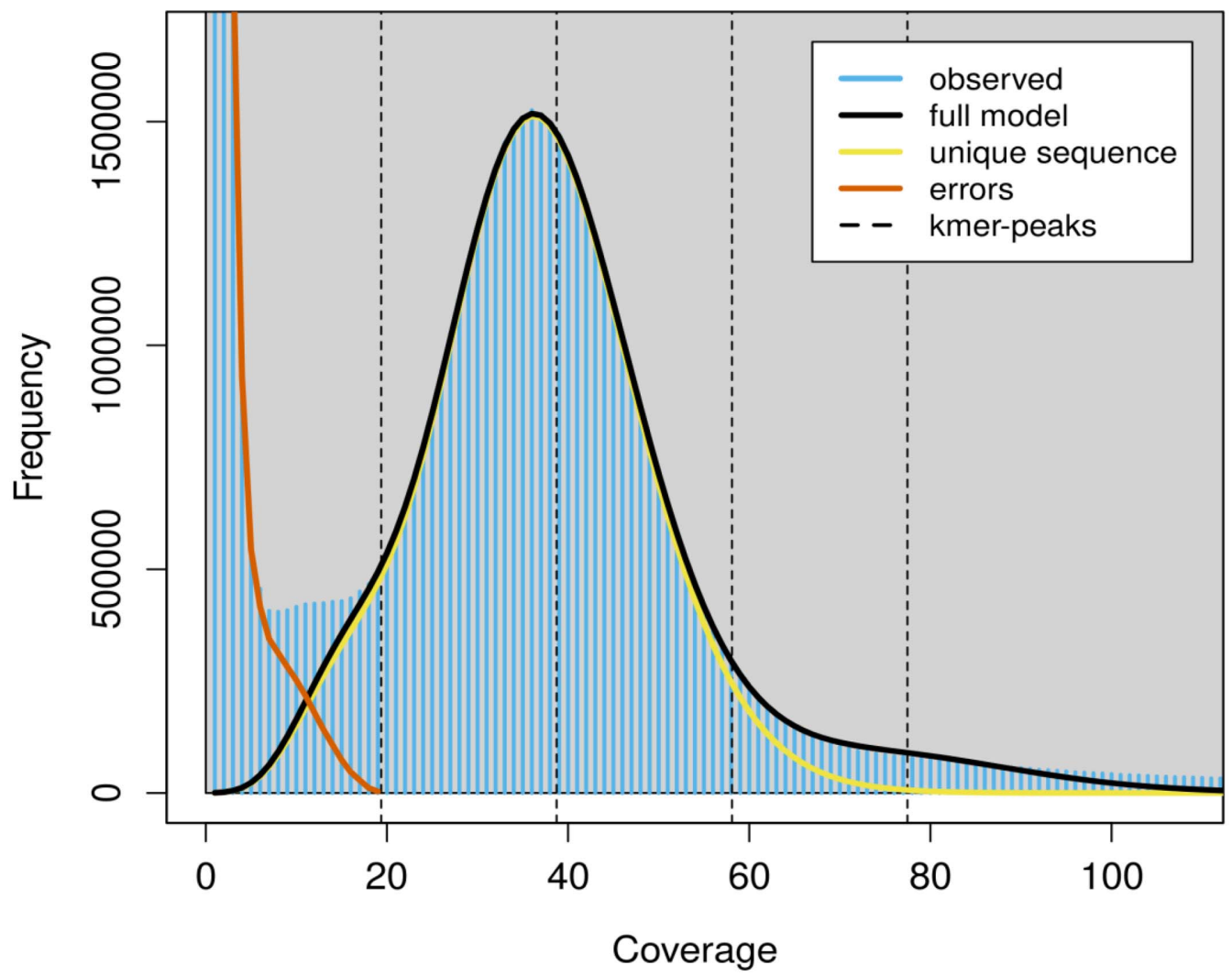

B Complete (C) and single-copy (S) Fragmented (F) Complete (C) and duplicated (D) Missing (M)

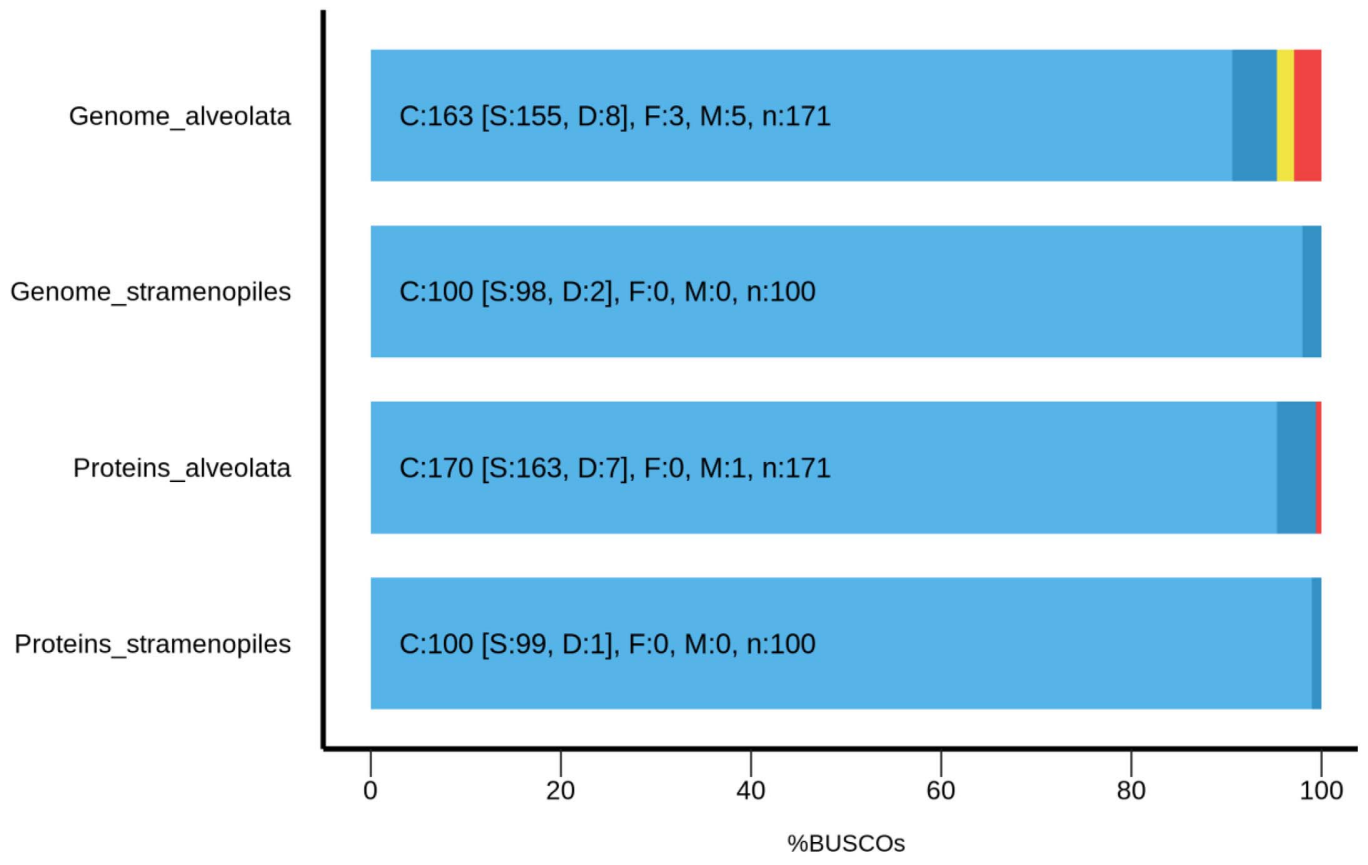

Fig. 1. Genome features of Phytophthora colocasiae strain Lyd2019. A, Genome size, repeat, and heterozygosity were estimated by GenomeScope ver. 2.0 using Illumina short reads. B, Completeness of genome assembly and predicted genes evaluated by BUSCO ver. 5.0 .0 with alveolata and stramenopiles datasets. Note: Completeness of genome predicted genes was evaluated with translated proteins using BUSCO under protein model. 
contigs with a contig $\mathrm{N}_{50}$ of $1.70 \mathrm{Mb}\left(\mathrm{L}_{50}=20\right)$, average contig length of $0.79 \mathrm{Mb}$, and maximum contig length of $3.93 \mathrm{Mb}$ (Table 1). The assembly size was very similar to the estimated genome size (92.51 versus $92.30 \mathrm{Mb})$. Compared with the previously reported genome assembly of strain 7290 (Vetukuri et al. 2018), the genome size of our assembly was $63.45 \%$ larger (92. 51 versus $56.60 \mathrm{Mb}$ ), with $99.47 \%$ fewer contigs (105 versus 19,853) and an approximately 1,382-fold larger contig $\mathrm{N}_{50}$ value $(1.70 \mathrm{Mb}$ versus $1.23 \mathrm{~kb})$. These findings indicate that high-level repeats may result in high fragmentation in short-read-based genome assembly, which can be corrected through long-read sequencing.

Genome completeness was assessed using BUSCO ver. 5.0.0 software (Seppey et al. 2019) (https://busco.ezlab.org/) with alveolata $(n=171)$ and stramenopiles datasets $(n=$ 100). The assembly was found to contain $95.32 \%$ (163 of 171) and $100 \%$ (100 of 100 ) complete single-copy orthologs at alveolata and stramenopiles level, respectively (Fig. 1B). The Illumina short reads were mapped to the genome assembly, and the mapping rate was used to evaluate genome completeness; we found that $96.67 \%$ of the reads were properly mapped (Table 1). These results provide solid evidence that our assembly had high continuity and completeness.

We further generated a library of de novo repeats using RepeatModeler ver. 2.01 (http:// www.repeatmasker.org/RepeatModeler/), and used it to perform repeat masking with RepeatMasker ver. 4.1.1 (http://www.repeatmasker.org/). We identified 52.78\% of our genome assembly as repetitive sequences (Table 1), consistent with the estimated repeat content $(56.71 \%)$. BRAKER2 (Hoff et al. 2019) and Fgenesh (Solovyev et al. 2006) were then applied for protein-coding gene (PCG) prediction using the repeat-masked genome assembly; this process integrated ab initio and homology-based gene prediction using proteins from Peronophythora sojae as a training dataset. In total, 18,322 PCGs were identified, among which 12,782 were annotated by InterProScan ver. 5.47 .82 (Jones et al. 2014) or eggNOG mapper ver. 2 (Huerta-Cepas et al. 2019), using the Pfam (11,538; 63.22\%), Gene Ontology (GO; 4,375; 23.88\%), Kyoto Encyclopedia of Genes and Genomes (KEGG; 6,857; 37.42\%), Eukaryotic Orthologous Groups (KOG; 11,382; 62.12\%), and carbohydrate-active enzymes (CAZys; $335 ; 1.8 \%$ ) databases (Table 1). Proteins with signal peptide (signalP ver. 5 ) and without transmembrane helices (TMHMM ver. 2.0) in mature protein were defined as candidate effectors in this study. We found 191 candidate RXLR effectors (with RxLR motif) and only 1 candidate crinkling and necrosis (CRN) effector (with LxLFLAK-HVLVxxP motif) (Table 1). We also try to find more candidate $\mathrm{CRN}$ effectors with similarity search by Pfam (no one hit with crinkler effector protein N-terminal domain PF20147) and eggNOG mapper (93 annotated with CRN family protein). However, only three of those with signal peptide but without CRN motif were defined as candidate CRN-like effectors.

Completeness of predicted genes were also evaluated by BUSCO with a protein model, and predicted genes contained 170 complete single-copy orthologs (including 7 duplicated) in the alveolata dataset and 100 complete single-copy orthologs (including 1 duplicated) in the stramenopiles dataset (Fig. 1B).

The whole-genome sequence data as well as the gene prediction and annotation information reported in this article has been deposited in the Genome Warehouse (https://ngdc.cncb. ac.cn/gwh/) in the National Genomics Data Center, Beijing Institute of Genomics (China National Center for Bioinformation), Chinese Academy of Sciences, under accession number GWHAOTV00000000 (BioProject: PRJCA003784). This Whole Genome Shotgun project has also been deposited at NCBI under the accession JAGKQI000000000.

\section{Literature Cited}

Hoff, K. J., Lomsadze, A., Borodovsky, M., and Stanke, M. 2019. Whole-genome annotation with BRAKER. Methods Mol. Biol. 1962:65-95.

Huerta-Cepas, J., Szklarczyk, D., Heller, D., Hernández-Plaza, A., Forslund, S. K., Cook, H., Mende, D. R., Letunic, I., Rattei, T., Jensen, L. J., von Mering, C., and Bork, P. 2019. eggNOG 5.0: A hierarchical, functionally and phylogenetically annotated orthology resource based on 5090 organisms and 2502 viruses. Nucleic Acids Res. 47:D309-D314.

Jackson, G. V. H., Gollifer, D. E., and Newhook, F. J. 1980. Studies on the taro leaf blight fungus Phytophthora colocasiae in the Solomon Islands: Control by fungicides and spacing. Ann. Appl. Biol. 96:1-10.
Jones, P., Binns, D., Chang, H. Y., Fraser, M., Li, W., McAnulla, C., McWilliam, H., Maslen, J., Mitchell, A., Nuka, G., Pesseat, S., Quinn, A. F., Sangrador-Vegas, A., Scheremetjew, M., Yong, S. Y., Lopez, R., and Hunter, S. 2014. InterProScan 5: Genome-scale protein function classification. Bioinformatics 30:12361240.

Kreike, C. M., Van Eck, H. J., and Lebot, V. 2004. Genetic diversity of taro, Colocasia esculenta (L.) Schott, in Southeast Asia and the Pacific. Theor. Appl. Genet. 109:761-768.

Lee, Y., Cho, K. S., Seo, J. H., Sohn, K. H., and Prokchorchik, M. 2020. Improved genome sequence and gene annotation resource for the potato late blight pathogen Phytophthora infestans. Mol. Plant-Microbe Interact. 33:1025-1028. 
Mbong, G. A., Fokunang, C. N., Lum, A., Fontem, B. M., and Tembe, E. A. 2013. An overview of Phytophthora colocasiae of cocoyams: A potential economic disease of food security in Cameroon. Discourse J. Agric. Food Sci. 1:140-145.

Mbong, G. A., Fokunang, C. N., Manju, E. B., Njukeng, A. P., Tembe-Fokunang, E. A., and Itanna, R. 2015. Mycelia growth and sporulation of Phytophthora colocasiae isolates under selected conditions. Am. J. Exp. Agric. 8:193-201.

Mishra, A. K., Sharma, K., and Misra, R. S. 2008. Effect of benzyl amino purine on the pathogen growth and disease development of taro leaf blight caused by Phytophthora colocasiae. J. Plant Pathol. 90:191-196.

Raciborski, M. 1900. Parasitic algae and fungi, java. Batavia Bull. N. Y. State Mus. 19:189.

Ranallo-Benavidez, T. R., Jaron, K. S., and Schatz, M. C. 2020. GenomeScope 2.0 and Smudgeplot for reference-free profiling of polyploid genomes. Nat. Commun. 11:1432.

Seppey, M., Manni, M., and Zdobnov, E. M. 2019. BUSCO: Assessing genome assembly and annotation completeness. Methods Mol. Biol. 1962:227-245.
Singh, D., Jackson, G., Hunter, D., Fullerton, R., Lebot, V., Taylor, M., Losefa, T., Okpul, T., and Tyson, J. 2012. Taro leaf blight-A threat to food security. Agriculture 2:182-203.

Solovyev, V., Kosarev, P., Seledsov, I., and Vorobyev, D. 2006. Automatic annotation of eukaryotic genes, pseudogenes and promoters. Genome Biol. 7:S10.

Srivastava, S. K., Abad, Z. G., Knight, L. M., Zeller, K., Mavrodieva, V., and Nakhla, M. 2020. Draft genome resource for the ex-types of Phytophthora ramorum, $P$. kernoviae, and $P$. melonis, species of regulatory concern, using ultralong read minION nanopore sequencing. Mol. Plant-Microbe Interact. 33:794797.

Vasquez, E. A. 1990. Yield loss in taro due to Phytophthora leaf blight. J. Root Crops 16:48-50.

Vetukuri, R. R., Kushwaha, S. K., Sen, D., Whisson, S. C., Lamour, K. H., and Grenville-Briggis, L. J. 2018. Genome sequence resource for the oomycete taro pathogen Phytophthora colocasiae. Mol. Plant-Microbe Interact. 31:903-905. 